\title{
THE MICROWAVE SPECTRUM OF ARGON-PHOSPHORUS TRIFLUORIDE
}

\author{
Kurt W. HILLIG II, Jose MATOS, Anthony SCIOLY and Robert L. KUCZKOWSKI \\ Department of Chemistry. University of Michigan. Ann Arbor. MI 48109. USA
}

Received 21 October 1986

\begin{abstract}
The argon- $\mathrm{PF}_{3}$ complex has been prepared in a supcrsonic expansion of $\mathrm{Ar}(98 \%)$ and $\mathrm{PF}_{3}(2 \%)$. A Fourier-transform microwave spectrometer employing a Fabry-Pérot cavity was used to assign 28 rotational transitions. The rotational constants ( $\mathrm{MHz}$ ) and distortion constants $(\mathrm{kHz})$ were $A=7332.468(10), B=1023.055(2), C=952.564(2), D_{J}=3.53(1), D_{J K}=60.4(1)$ and $d_{1}=-0.240(7)$. The argon atom is $3.953 \AA\left(r_{\mathrm{cm}}\right)$ from the $\mathrm{PF}_{3}$ center of mass and $r_{\mathrm{cm}}$ makes an angle of $70.3^{\circ}$ with the $C_{3}$ axis of the $\mathrm{PF}_{3}$.
\end{abstract}

\section{Introduction}

Several van der Waals complexes with a high atom percentage of fluorine have been studied by high-resolution techniques. Examples include $\mathrm{BF}_{3}-\mathrm{N}_{2}[1]$, $\mathrm{HCF}_{3}$ bonded to an acid or ammonia [2,3], $\mathrm{COF}_{2}-\mathrm{Ar}$ [4], ClF-Ar [5], and a number of HF complexes [6]. Most of the systems are linear or symmetric tops. Usually the fluorine atoms are distant from the second species and do not appear to directly interact with it. $\mathrm{Ar}-\mathrm{COF}_{2}$ is an exception; it is an asymmetric top (of necessity) with the Ar located above the $\mathrm{CF}_{2}$ triangle. We report here the microwave spectrum of the Ar- $\mathrm{PF}_{3}$ complex which is also an asymmetric top. In this complex, the fluorine is closer than phosphorus to the argon atom.

\section{Experimental}

The spectrum was observed with a Fourier-transform microwave spectrometer of the Balle-Flygare type [7] with a pulsed nozzle gas source. The spectrometer operated in the region of $8-18 \mathrm{GHz}$ with a HP $8671 \mathrm{~B}$ synthesizer as the MO. The microwave cavity employed aluminum mirrors with a diameter of $35 \mathrm{~cm}$ and radius of curvature of $85 \mathrm{~cm}$ separated by $\approx 60 \mathrm{~cm}$. A semicircular wire loop ( $\approx 1 \mathrm{~cm}$ diameter) was used as the transmitting and receiving antenna. Heterodyne techniques converted the coherent MW emission to a low-frequency signal which was sampled at 1024 points at 200 or 500 ns intervals by a Datalab DL910 transient recorder (eightbit resolution). The FID signal was transferred to a PDP-1 1/23 computer where signal averaging of up to 100000 shots and the Fourier transformation were carried out. A Newport BV-100 pulsed nozzle and driver were used with a pulse repetition rate of about $6 \mathrm{~Hz}$ (limited by the data transfer rate to the computer). The nozzle orifices were 0.5 and $1.0 \mathrm{~mm}$ and the backing pressure was typically $1-2 \mathrm{~atm}(\approx 98 \%$ Ar, $2 \% \mathrm{PF}_{3}$ ). Doppler splitting of the lines [8] was minimized by controlling the pulse timing to examine the leading edge of the gas pulse entering the cavity. Typical linewidths of $\approx 8 \mathrm{kHz}$ were observed except in some very-low- $J$ transition where hyperfine interactions appeared to complicate and broaden the line shape.

\section{Spectra and analysis}

The first transitions were observed in a spectral region predicted for a symmetric top $\mathrm{Ar}-\mathrm{PF}_{3}$ complex. Gas mixing and purity tests indicated that both $\mathrm{Ar}$ and $\mathrm{PF}_{3}$ were essential for observation of the spectrum. However, subsequent searches could not identify the additional transitions expected for a 
Table 1

Observed and calculated rotational transitions for $\mathrm{Ar}_{-} \mathrm{PF}_{3}(\mathrm{MHz})$

\begin{tabular}{|c|c|c|c|c|}
\hline$J^{\prime}\left(K_{\mathrm{p}}^{\prime}, K_{0}^{\prime}\right)$ & $J\left(K_{\mathrm{p}}, K_{0}\right)$ & $\begin{array}{l}\text { Freq. } \\
\text { (obs.) }\end{array}$ & $\begin{array}{l}\text { Freq. } \\
\text { (calc.) }\end{array}$ & Calc. - obs. \\
\hline $1(1,0)$ & $0(0,0)$ & 8355.380 & 8355.387 & 0.007 \\
\hline $2(1,1)$ & $1(0,1)$ & 10401.150 & 10401.150 & 0.000 \\
\hline $3(1,2)$ & $2(0,2)$ & 12482.069 & 12482.068 & -0.001 \\
\hline $4(1,3)$ & $3(0,3)$ & 14598.771 & 14598.768 & -0.003 \\
\hline $5(1,4)$ & $4(0,4)$ & 16752.300 & 16752.299 & -0.001 \\
\hline $8(0,8)$ & $7(1,6)$ & 8364.500 & 8364.499 & -0.001 \\
\hline $9(0,9)$ & $8(1,7)$ & 9999.103 & 9999.103 & 0.000 \\
\hline $10(0,10)$ & $9(1,8)$ & 11578.049 & 11578.051 & 0.002 \\
\hline $4(0,4)$ & $3(0,3)$ & 7895.704 & 7895.705 & 0.001 \\
\hline $4(1,3)$ & $3(1,2)$ & 8040.831 & 8040.828 & -0.003 \\
\hline $4(2,3)$ & $3(2,2)$ & 7899.198 & 7899.185 & -0.013 \\
\hline $4(2,2)$ & $3(2,1)$ & 7905.048 & 7905.054 & 0.006 \\
\hline $5(0,5)$ & $4(0,4)$ & 9864.608 & 9864.605 & -0.003 \\
\hline $5(1,5)$ & $4(1,4)$ & 9697.088 & 9697.085 & -0.003 \\
\hline $5(1,4)$ & $4(1,3)$ & 10049.236 & 10049.236 & -0.000 \\
\hline $5(2,4)$ & $4(2,3)$ & 9872.633 & 9872.613 & -0.020 \\
\hline $5(2,3)$ & $4(2,2)$ & 9884.323 & 9884.340 & 0.017 \\
\hline $5(3,3)$ & $4(3,2)$ & 9872.875 & 9872.885 & 0.010 \\
\hline $5(3,2)$ & $4(3,1)$ & 9872.940 & 9872.949 & 0.009 \\
\hline $6(0,6)$ & $5(0,5)$ & 11830.173 & 11830.175 & 0.002 \\
\hline $6(1,6)$ & $5(1,5)$ & 11634.069 & 11634.068 & -0.001 \\
\hline $6(1,5)$ & $5(1,4)$ & 12056.425 & 12056.428 & 0.003 \\
\hline $6(2,5)$ & $5(2,4)$ & 11845.155 & 11845.129 & -0.026 \\
\hline $6(2,4)$ & $5(2,3)$ & 11865.582 & 11865.622 & 0.040 \\
\hline $7(0,7)$ & $6(0,6)$ & 13791.773 & 13791.779 & 0.006 \\
\hline $7(1,7)$ & $6(1,6)$ & 13569.750 & 13569.752 & 0.002 \\
\hline $7(1,6)$ & $6(1,5)$ & 14062.136 & 14062.146 & 0.010 \\
\hline $7(2,6)$ & $6(2,5)$ & 13816.587 & 13816.552 & -0.035 \\
\hline
\end{tabular}

symmetric top species. Asymmetric models with the Ar swinging $180^{\circ}$ between the two ends of the $\mathrm{PF}_{3}$ molecule were then examined and led to a successful search for confirmatory transitions. A total of $28 \mu_{a}$ and $\mu_{c}$ transitions were assigned. They are listed in table 1 . The derived rotational constants and centrifugal distortion constants are given in table 2 . The $D_{J}$ and $D_{J_{K}}$ constants are approximately 2-3 times smaller than for the Ar-COF ${ }_{2}$ species [4]. No evidence for internal rotation of the $\mathrm{PF}_{3}$ was observed.

Table 2

Spectroscnnic constants for $\mathrm{Ar}-\mathrm{PF}_{3}{ }^{\text {a) }}$

$$
\begin{array}{ll}
A=7332.468(10) \mathrm{MHz} & D_{J}=3.53(1) \mathrm{kHz} \\
B=1023.055(2) \mathrm{MHz} & D_{J K}=60.4(1) \mathrm{kHz} \\
C=952.564(2) \mathrm{MHz} & d_{1}=-0.240(7) \mathrm{kHz}
\end{array}
$$

\footnotetext{
a) Distortion constants from van Eijk-Typke-Watson $S$-reduction [9], representation $I^{r}$.
}

Transition intensities for $J=7$ and $J=8$ levels $(E \approx 2$ $\mathrm{cm}^{-1}$ ) began to fall off presumably due to the low rotational temperature in the molecular beam. Hyperfine structure was partially resolvable for several low- $J$ transitions $(J \leqslant 2)$ due to the presence of phosphorus $(I=1 / 2)$ and two kinds of fluorine atoms $(I=1 / 2)$. The complexity of the coupling precluded an analysis at this stage.

The value of $I_{a}+I_{c}-I_{b}=105.3186 \mathrm{amu} \AA^{2}$ is close to the value expected $\left(105.3027 \mathrm{amu} \AA^{2}\right)$ for two fluorine atoms straddling a plane of symmetry based on the structure of free $\mathrm{PF}_{3}[10]$. The discrepancy between the observed and ideal value is smaller than often observed for van der Waals complexes where large-amplitude vibrational motions usually contribute more noticeable vibration-rotation interaction effects. With the assumption of a plane of symmetry and no change in $\mathrm{PF}_{3}$ structural parameters upon 
A
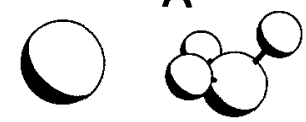

C
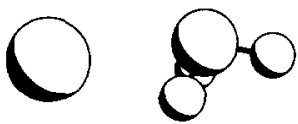

B
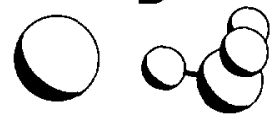

D

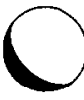

Fig. 1. Conformations of $\mathrm{Ar}-\mathrm{PF}_{3}$ which are compatible with the observed rotational constants.

adduct formation, two additional parameters can be determined, viz. the distance of the argon atom from the center of mass of the $\mathrm{PF}_{3}$ moiety $\left(r_{\mathrm{c.m}}\right.$. $)$ and the angle $(\theta)$ between $r_{\text {c.m }}$ and the $\mathrm{PF}_{3}$ symmetry axis. The program STRFIT originally written by Schwendeman [10] and revised by us was used to analyze the moments of inertia. The resultant fit $\left(\Delta I_{\text {calc }}-\Delta I_{\text {obs }}=0.0709 \mathrm{amu} \AA^{2}\right)$ gave $\theta=70.3^{\circ}$ and $r_{\mathrm{cm}}=3.9533 \AA$ A . Because of the symmetry of $\mathrm{PF}_{3}$, its orientation relative to the $\mathrm{Ar}$ is ambiguous. The four conformations which are consistent with the data are illustrated in fig. 1. Conformation $A$ is transformed into $\mathrm{B}$ by a rotation of $180^{\circ}$ (or $60^{\circ}$ ) about the $C_{3}$ axis of the $\mathrm{PF}_{3}$. Conformations $\mathrm{C}$ and $\mathrm{D}$ result by inverting the $\mathrm{PF}_{3}$ in $\mathrm{A}$ and $\mathrm{B}$ through its center of mass.

It is interesting that the four structures each suggest a different interaction. The Ar appears to interact with two fluorines in $\mathrm{A}$, with one fluorine in $\mathrm{B}$, with a $\mathrm{PF}_{2}$ face in $\mathrm{C}$ and roughly with a $\mathrm{PF}$ bond in $D$. Conformation $B$ is considered unlikely since the Ar-F distance is $2.590 \AA$ or about $0.75 \AA$ shorter than the sum of the van der Waals radii. Conformation $D$ also has a somewhat short Ar-F distance (2.856 $\AA$ ) although this is not so clearly an implausible value. In conformations $\mathrm{A}$ and $\mathrm{C}$ the $\mathrm{Ar}-\mathrm{F}$ distance is 3.430 and $3.636 \AA$, respectively. The Ar-P distance is 4.149 $\AA$ in $A$ and $B$ and $3.814 \AA$ in $C$ and D. It is not possible to resolve the structural ambiguity by investigating additional isotopic species. Phosphorus and fluorine have only one stable isotope and ${ }^{36} \mathrm{Ar}$ substitution would not provide any additional structural information even if it could be observed.
Preliminary ab initio fixed geometry calculations [11] at the 6-21G level with second-order Møller-Plesset corrections (MP2) to the correlation energy gave the following relative energy ordering: $\mathrm{A}=0 \mathrm{~cm}^{-1}, \mathrm{C}=109 \mathrm{~cm}^{-1}, \mathrm{D}=206 \mathrm{~cm}^{-1}, \mathrm{~B}=878$ $\mathrm{cm}^{-1}$. Only conformation $A$ was bound (total energy $\approx 80 \mathrm{~cm}^{-1}$ less than the sum of the Ar and $\mathrm{PF}_{3}$ energies). It is difficult to judge the usefulness of this approach apart from another indicator that B is implausible. The calculated energy differences between the other three conformers are not large and small changes in geometries could alter the ordering. More work is planned to explore this question.

This study demonstrates that argon forms a weak complex with $\mathrm{PF}_{3}$ in which fluorine is closer than phosphorus to the argon. In contrast, Ar binds to the more positive species [12] in the linear $\mathrm{Ar}-\mathrm{Cl}-\mathrm{F}$ complex. One resolution of this paradox arises from application of the proposal that van der Waals molecules mimic the structures of their isoelectronic chemical analogs $[5,13]$. This would suggest that conformation $\mathrm{C}$ with the phosphorus lone pair and two fluorines in pseudoequatorial positions of a trigonal bipyramid is the preferred arrangement. We intend to examine the $\mathrm{Ar}-\mathrm{PF}_{2} \mathrm{Cl}$ complex as a means to further explore this question.

\section{Acknowledgement}

We gratefully acknowledge the considerable assistance of Mr. David Crosby of the University of Michigan Physics Department and Professor Valdis Liepa of the Electrical Engineering Department with the design and trouble-shooting of the spectrometer. We acknowledge the help of Professor H.D. Rudolph and Dr. V. Typke of Ulm University who provided the ZFAP centrifugal distortion program used to fit the spectrum. The work was supported by Grant CHE-8305806 from the National Science Foundation.

\section{References}

[1] K.C. Janda, L.S. Bernstein, J.M. Steed, S.E. Novick and W. Klemperer, J. Am. Chem. Soc. 100 (1978) 8074

[2] G.T. Fraser, F.J. Lovas, R.D. Suenram, D.D. Nelson Jr. and W. Klemperer, J. Chem. Phys. 84 (1986) 5983. 
[3] E.J. Goodwin and A.C. Legon, J. Chem. Phys. 84 (1986) 1988.

[4] J.A. Shea and E.J. Campbell, J. Chem. Phys. 79 (1983) 4724.

[5] S.J. Harris, S.E. Novick and W. Klemperer, J. Chem. Phys. $61(1974) 193$.

[6] A.C. Legon and D.J. Miller, Chem. Rev. 86 (1986) 635.

[7] T.J. Balle and W.H. Flygare, Rev. Sci. Instr. 52 (1981) 33.

[8] E.J. Campbell, L.W. Buxton, T.J. Balle, M.R. Keenan and W.H. Flygare, J. Chem. Phys. 74 (1981) 829.

[9] W. Gordy and R.L. Cook, Microwave molecular spectra (Wiley, New York, 1984) p. 333.
[10] R.H. Schwendeman, in: Critical evaluation of chemical and physical structural information, eds. D.R. Lide and M.A. Paul (National Academy of Sciences, Washington, 1974) pp. 74-115.

[11] W.J. Hehre, L. Radom, P. von R. Schleyer and J.A. Pople, Ab initio molecular orbital theory (Wiley, New York, 1986).

[12] B. Fabricant and J.S. Muenter, J. Chem. Phys. 66 (1977) 5274.

[13] S.J. Harris, K.C. Janda, S.E. Novick and W. Klemperer, J. Chem. Phys. 63 (1975) 881. 